Chamorro Guerrero, M. ${ }^{a}$ Dolores (2018). «Las colocaciones en el español académico: diseño de una investigación experimental sobre los efectos del Enfoque Léxico», en RILEX. Revista sobre investigaciones léxicas, 1/I, pp. 113-134.

\title{
LAS COLOCACIONES EN EL ESPAÑOL ACADÉMICO: DISEÑO DE UNA INVESTIGACIÓN EXPERIMENTAL SOBRE LOS EFECTOS DEL ENFOQUE LÉXICO
}

\section{COLLOCATION IN ACADEMIC SPANISH: AN EXPERIMENTAL RESEARCH PROJECT ON THE EFFECTS OF THE LEXICAL APPROACH}

\author{
M. ${ }^{a}$ Dolores Chamorro Guerrero \\ Centro de Lenguas Modernas \\ Universidad de Granada \\ lola.chamorroguerrero@yahoo.es
}

\begin{abstract}
RESUMEN
Desde hace ya alguna década, distintos estudios neurolingüísticos vienen subrayando la importancia de los elementos prefabricados en la construcción del discurso oral y escrito de los hablantes nativos. Esta orientación de la investigación ha abierto interesantes perspectivas para el desarrollo de muchas áreas lingǘsticas, y en concreto para la pedagogía de lenguas, pues parece que se allana el camino para que el aprendiz de una lengua se haga con un dominio más efectivo de ella, como sostiene el Enfoque Léxico. Desgraciadamente no existen suficientes estudios empíricos que validen los presupuestos de esta metodología. Como contribución preliminar para subsanar esta carencia, presentamos en este artículo una propuesta de investigación experimental consistente en la elaboración de un glosario de colocaciones, que permita desarrollar una herramienta didáctica en base a la cual pueda evaluarse el efecto de la instrucción explícita de las colocaciones en la producción de textos en el ámbito académico.
\end{abstract}

Palabras clave: Lexicología, Lingüística aplicada, Enseñanza de lenguas, Español LE

\begin{abstract}
For some decades now, various neurolinguistic studies have highlighted the importance of prefabricated elements in the construction of oral and written discourse among native speakers. This line of research has lead to the development of new and interesting perspectives within many linguistic areas, not least in the field of language teaching, in which theories such as the Lexical Approach appear to be paving the way towards the more effective achievement of second language proficiency. Unfortunately, empirical studies that validate this methodological approach are still lacking. The present article attempts to address this issue, to some extent, by presenting an experimental research proposal focused on the elaboration of a glossary of collocations. This glossary will facilitate the development of a didactic tool through which the effect of explicit instruction of collocations in the production of academic texts can be evaluated.
\end{abstract}

Keywords: Lexicology, Applied Linguistics, Language Teaching, Spanish SL 


\section{INT'RODUCCIÓN}

Frente a lo que ha ocurrido con otras áreas de estudio sobre la adquisición de la lengua, la investigación sobre el vocabulario ha estado muy próxima a los contextos de enseñanza. A principios de los noventa toma cuerpo una tendencia pedagógica, el llamado Enfoque Léxico (Lewis, 1993), que aúna, de una parte, los resultados de estudios recientes sobre el léxico a partir de la observación de los hablantes nativos y, de otra, las experiencias de los profesores en su actividad en las aulas cuando aplican de manera más o menos consecuente las distintas metodologías de enseñanza tras la consolidación del método comunicativo.

Por eso, uno de los aspectos más sugerentes de la propuesta del Enfoque Léxico es que no se limita a ofrecer alternativas teóricas sobre posibles modos de reorientar la enseñanza, sino que propone actuaciones concretas, bien fundamentadas y coherentes con su visión de la enseñanza, ajustada a las necesidades de alumnos y profesores.

Recordemos los presupuestos principales de esta metodología, enunciados por Lewis $(1993,1997,2000)$. En ella se pone el énfasis en desarrollar la competencia léxica de los aprendices, pues se considera esencial para que alcancen la competencia comunicativa general. La conocida cita de Wilkins (1972), con la que se iniciaba décadas antes el método nociofuncional, recoge bien la esencia del Enfoque Léxico: "Sin gramática poco puede ser expresado, sin vocabulario nada". Así pues, el léxico se coloca en el centro de la práctica didáctica, que se dirige a mejorar la competencia léxica, entendida esta como el dominio de un número amplio de unidades léxicas; el conocimiento de sus diferentes tipos, de las combinaciones y restricciones combinatorias de las palabras y el reconocimiento de su valor pragmático. Solo el contacto reiterado con un input rico permitirá al aprendiz acceder a dicho conocimiento, por lo que el enfoque considera primordial fomentar en el aula la práctica de las destrezas receptivas, en especial de la comprensión oral, pues es en el discurso nativo donde los aprendices pueden identificar patrones léxicos con los que componer su propio discurso. De este modo, Lewis (1993) recoge la idea procedente de estudios neurolingüísticos de la importancia de los elementos prefabricados en la elaboración del discurso y propone desarrollar la conciencia lingüística del aprendiz 
para que pueda identificar dichos prefabricados e incorporarlos a su bagaje. Para este autor la lengua debe considerarse léxico gramaticalizado y no gramática lexicalizada. Esta visión de la lengua condiciona enormemente su idea de la enseñanza. A su modo de ver, esta debe centrarse en hacer evidentes los patrones léxicos, cuya adecuación está garantizada, al ser lo que los hablantes de una lengua usan, mientras que los patrones estructurales son tan solo útiles como referencia para que los aprendices construyan sus enunciados. Finalmente Lewis llega incluso a afirmar que la fluidez que un aprendiz consiga en una lengua va a depender de su habilidad para unir segmentos de lengua de un modo conveniente.

Con el tiempo, y no sin algunas reticencias desde algunos sectores de la enseñanza, el Enfoque Léxico ha ido ganando adeptos entre profesores y alumnos ya que estos últimos sienten que les facilita una comunicación más espontánea. En efecto, como sostiene este enfoque, al acceder a patrones léxicos —elementos prefabricadosapropiados a distintas situaciones, el aprendiz consigue un discurso más fluido y más adecuado a sus necesidades de expresión, lo que tiene efectos muy positivos en su motivación, y por lo tanto en la de los profesores, que ven desarrollarse su labor con mayor productividad.

Sin embargo, más allá de la percepción que se pueda tener de la aplicación del Enfoque Léxico en el aula, aún no tenemos la total certeza de que esta suponga una mejora empíricamente mensurable en el dominio de la nueva lengua por parte de los aprendices. Ciertamente faltan aún instrumentos que nos puedan proporcionar evidencias de su rentabilidad, pues no existen suficientes estudios empíricos sobre el efecto de esta metodología en el aprendizaje. De ahí que desde la teoría de adquisición se estén reclamando investigaciones que sustenten experimentalmente las afirmaciones del enfoque.

De hecho, en palabras de una de las más reputadas especialistas en este campo, Higueras, este es uno de los retos que, tras muchos logros, le quedan por afrontar a los defensores de esta perspectiva metodológica, validar su eficacia "ya que la investigación en una disciplina aplicada como la enseñanza de lenguas debe partir del aula y volver al aula" (Higueras, 2017b, p.15). Esta es la dirección que queremos darle a nuestro 
trabajo presentando en él una propuesta centrada en el tema de las colocaciones en el español académico, que se perfila como una de las carencias que los estudiantes más interés tienen en subsanar.

\section{DESARROLLO DE LA IDEA DE COLOCACIÓN}

Como ya se ha mencionado, una de las mayores aportaciones del Enfoque Léxico a los estudios lingüísticos ha sido la de señalar la necesidad de atender en el estudio del vocabulario a diferentes unidades léxicas, segmentos prefabricados de distinta extensión y con distintos grados de idiomaticidad —fórmulas rutinarias, colocaciones, compuestos sintagmáticos y locuciones idiomáticas, en la terminología de Gómez Molina (2004b) — Como resultado de ello se han venido realizando muchos trabajos dirigidos a identificar y analizar el comportamiento sintáctico y los valores semánticos y pragmáticos de dichas unidades. Una atención especial ha recibido en la investigación sobre adquisición y pedagogía de segundas lenguas el estudio de los paquetes léxicos (lexical bundles o clusters) (Biber et al., 1999; Hyland, 2008a) y de las colocaciones (Schmitt, 2004; Wood, 2010 y Lewis, 2000; Thornbury, 2002; Boers et al. 2006). En español el interés por las unidades colocacionales ha llegado tardíamente: hasta los años 2000 no aparecen los primeros estudios descriptivos (Koike, 2001), lexicográficos —los diccionarios combinatorios DICE (Alonso Ramos, 2004) y REDES (Bosque, 2004)— y los orientados a la didáctica del español como lengua extranjera (Higueras, 2004, 2006a; Ferrando, 2010).

Curiosamente, entre dos disciplinas tan próximas como la Lingüística y la Didáctica de lenguas se producen divergencias en la forma de considerar el concepto de colocación. Para la primera de estas disciplinas (y siempre hablando de una forma general), las colocaciones son combinaciones restringidas de al menos dos palabras, que presentan una serie de rasgos formales y semánticos, como son la coocurrencia frecuente, la direccionalidad, la tipicidad de la relación, la fijación arbitraria en la norma, 
la regularidad sintáctica y la transparencia semántica ${ }^{1}$. En este sentido se toman por colocaciones combinaciones como enarcar las cejas, albergar esperanzas o momento crucial.

La Didáctica de lenguas extranjeras trabaja con una idea más laxa de colocación, considerándola como una combinación de palabras que suelen aparecer juntas y que muestran un cierto grado de fijación léxica en el uso de los hablantes de una lengua. Así, se reconocen como colocaciones expresiones como bacer la cama, echar una siesta o comida casera. Algunas de estas colocaciones no serían aceptadas como tales por la Lingüística, que las consideraría como combinaciones libres, del tipo leer un libro o tomar un café, en las que no se da la selección restrictiva de un componente de la combinación (base) sobre el otro (colocativo).

En este trabajo nos situaremos en la posición didáctica, para la que no es tan relevante atender exhaustivamente a los rasgos formales de la colocación que señala la Lingüística, ya que en nuestro caso resulta más significativo dirigir la atención de los aprendices a aquellas combinaciones que les serán más rentables para su producción de textos académicos, se trate o no de colocaciones prototípicas ${ }^{2}$. Es más, la experiencia en el aula nos dice que, en el desarrollo de estrategias de reconocimiento sobre patrones léxicos, la percepción de los aprendices, una vez que hemos enfocado su mirada hacia estos segmentos de lengua, se dirige asimismo a otras unidades pluriverbales, cuya utilidad es evidente para ellos. Así se detienen tanto en paquetes léxicos (no cabe duda de que) como en expresiones idiomáticas (partir de cero) o locuciones sintagmáticas (tener lugar). Es cierto que, a efectos de sistematización a la hora de llevar a cabo el diseño de las actividades, puede tener sentido establecer una diferencia entre ellas, dado que facilitan la selección y organización de los segmentos léxicos que se incluyen en la programación didáctica, pero no así para la implementación de este

\footnotetext{
1 Para el estudio del concepto de colocación, véase Corpas (1996); Castillo Carballo (1998); Lewis (2000); Koike (2001); Zuloaga (2002) e Higueras (2006a).

2 En esta línea se sitúa Higueras (2006a), quien subraya la importancia de las colocaciones no prototípicas en la enseñanza, por su elevado número y su frecuencia en los discursos nativos. Esta autora amplía el concepto de colocación que se maneja en los estudios lingüísticos teóricos y aboga por situar en un espectro los distintos agrupamientos léxicos, en lugar de reducirse a una clasificación discreta de estas unidades en colocaciones prototípicas y no prototípicas. Así señala: "Para nosotros las colocaciones sintagmáticas o colocaciones no prototípicas se perfilarían como un estadio intermedio entre las combinaciones libres y las colocaciones" (entendidas estas en el sentido que les otorga la Lingüística) (Higueras, 2006a, p. 27)
} 
contenido en la clase. Otro tanto ocurre con la distinción entre colocaciones prototípicas y no prototípicas de la que venimos hablando.

Nuestro estudio centra su interés en la identificación de colocaciones rentables para la elaboración de textos académicos, con el fin de crear un glosario de unidades que estén a disposición de los aprendices. Este primer paso en la investigación tiene una motivación doble: de un lado, nos permite diseñar las secuencias didácticas siguiendo los presupuestos del Enfoque Léxico sobre la base de las unidades seleccionadas, así como elaborar el instrumento con el que medir la respuesta de los aprendices ante este tipo de instrucción. Por otro lado, este glosario constituye un excelente recurso tanto para los estudiantes universitarios no nativos como para que profesores y diseñadores de materiales pedagógicos desarrollen la enseñanza de la escritura académica, un área en la que, hasta donde sabemos, no contamos con trabajos lexicográficos muy ajustados a sus necesidades, a pesar de su gran utilidad.

Es bien sabido que el desarrollo de la Lingüística computacional y la Lingüística de corpus ha abierto posibilidades extraordinarias a los estudios dedicados al léxico, al manejar grandes cantidades de textos y ofrecer muestras reales del uso nativo de distintos registros y géneros. Los diccionarios combinatorios y los distintos corpus CREA (Corpus de referencia del español actual) o CORPES XXI (Corpus del español del siglo $X X I)$ se han convertido en materiales de referencia indispensable para toda investigación en este campo $^{3}$. Sin embargo, por lo que respecta al tema de las colocaciones en el ámbito académico, aunque se han realizado avances en la descripción de estas unidades en ciertos campos temáticos y en la enseñanza del español con fines específicos, son escasos los trabajos que se dedican a estudiar las colocaciones en un ámbito más amplio, como es el del vocabulario académico tomado

\footnotetext{
${ }^{3}$ Se están desarrollando asimismo corpus de textos producidos por aprendices de español en distintos contextos, que serán de extremada utilidad para identificar los aspectos que requieran de una enseñanza explícita del léxico al detectar las dificultades de expresión relacionadas con la falta de competencia colocacional de los aprendices y con la interferencia de la lengua materna. Véase por ejemplo el corpus Aprescrilov, sobre producciones de aprendices neerlandeses (dirigido por Buyse, 2011) y el que el grupo de investigación Anacor está realizando en la actualidad para la comparación de la adquisición de español e inglés.
} 
en sentido general. ${ }^{4}$ Resulta evidente que, al margen del vocabulario propio de cada área de conocimiento, muchos aspectos de los discursos formales son interdisciplinares, es decir, recogen aquello que tienen en común todos los discursos especializados. La importancia de dedicar la atención a este conjunto léxico, marcado por la intersección de los discursos académicos concretos, no necesita ser enfatizada y, como ya se ha indicado, este aspecto ocupará una posición destacada en la realización del presente trabajo.

\section{ANTECEDEN'TES ME'TODOLÓGICOS}

En la línea del estudio que planteamos aquí merece señalarse el trabajo de Pérez Serrano (2015). Esta investigadora examina la efectividad del Enfoque Léxico en la adquisición de las colocaciones frente a propuestas didácticas que no comparten los presupuestos de dicho enfoque, e incluso va un paso más allá al comparar los resultados de aprendizaje de distintas metodologías dentro del propio Enfoque Léxico o inspiradas en él.

Con su investigación Pérez Serrano pretende aportar evidencias empíricas en torno a la discusión que mantienen los defensores de la corriente léxica sobre la eficacia de diferentes formas de plantear la instrucción de las colocaciones léxicas. De un lado, algunos investigadores enfatizan la necesidad de fomentar la percepción y el reconocimiento de las colocaciones en los contextos discursivos en los que aparecen, atendiendo al hecho de que una de sus características es ser transparentes — no crean problemas de comprensión-, por lo que suelen pasar desapercibidas para los aprendices (Lewis, 1993, 1997, 2000). Otros estudiosos, sin embargo, sostienen que es preferible promover un aprendizaje explícito de colocaciones descontextualizadas (Laufer y Girsai, 2008; Peters, 2009, 2012), pues consideran que la adquisición del léxico a medio y largo plazo requiere una atención expresa a sus rasgos semánticos y pragmáticos y un cierto grado de encuentro del aprendiz con esas unidades.

\footnotetext{
${ }^{4}$ Existe para el inglés la AWL (Academic Word List), recopilada por Averil Coxhead en 2000. Es una base de 570 familias de palabras, extraídas de un corpus de 3 millones de palabras procedentes de textos elaborados en el entorno universitario. Sin embargo, este trabajo se restringe a unidades monoverbales, se mantiene en el nivel de la palabra aislada.
} 
En nuestro caso no nos interesa tanto mostrar las diferencias entre los diferentes tipos de instrucción dentro del propio Enfoque Léxico cuanto comprobar los resultados en términos de rentabilidad pedagógica de dicho enfoque frente a una enseñanza que no atiende al léxico en su dimensión combinatoria.

\section{OBJETIVO E HIPÓTESIS DE INVESTIGACIÓN}

Para concretar el objetivo de este trabajo que hemos ido anticipando en epígrafes anteriores, diremos que se persigue la evaluación del efecto de la instrucción basada en el Enfoque Léxico en la adquisición de las colocaciones y en la producción de textos académicos. Nuestra investigación propone dos hipótesis fundamentales:

1. El aprendizaje centrado en la instrucción explícita de las colocaciones mejora sustancialmente la calidad de los textos académicos elaborados por los aprendices en términos de precisión, adecuación y naturalidad.

2. La utilización del Enfoque Léxico en la instrucción incide positivamente en el proceso de generación de los textos académicos, en tanto que facilita el flujo de las ideas del aprendiz.

A la confirmación o refutación de estas hipótesis vamos a dedicar el desarrollo de la presente investigación.

\section{METODOLOGÍA Y PROCEDIMIENTO}

Para llevar a cabo el estudio empírico, haremos un seguimiento de dos grupos: un grupo experimental y un grupo de control. En ambos casos la instrucción abordará la creación de textos académicos y tendrá como punto de partida el mismo contenido lingüístico, confeccionado a partir de una selección de resúmenes de investigación de nuestro corpus y del glosario de colocaciones. En el grupo de control se seguirá una enseñanza tradicional de la escritura académica, se trabajará al nivel de la palabra y de las relaciones paradigmáticas (de sinonimia, antonimia, hiponimia, etc.) sin incidir en la dimensión combinatoria del léxico. En el grupo experimental la instrucción atenderá de manera explícita a las colocaciones, promoviendo que los alumnos las reconozcan en los textos que se les proponen como modelo y las reutilicen en la elaboración de sus producciones escritas. El progreso en el desarrollo de la competencia colocacional 
de los aprendices entre el momento previo a la instrucción y el posterior a ella será medido mediante dos pruebas diseñadas a tal efecto.

Como hemos comentado más arriba, antes de emprender la investigación experimental, es imprescindible abordar la creación de un glosario de colocaciones, para seguidamente elaborar el tratamiento pedagógico al que vamos a exponer al grupo experimental. Para ello debemos abordar las siguientes fases:

a) Recoger los datos procedentes de las muestras de hablantes no nativos (HNN);

b) Crear un corpus de textos de hablantes nativos $(\mathrm{HN})$;

c) Delimitar el objeto de estudio y la elaboración de un glosario de colocaciones relevantes;

d) Sistematizar las colocaciones seleccionadas;

e) Diseñar las secuencias didácticas, tanto del grupo experimental como del grupo de control;

f) Elaborar los instrumentos de medida: el pretest y postest que determinarán el progreso de los estudiantes tras el trabajo realizado durante la instrucción;

g) Analizar y discutir los resultados, que llevarán a las conclusiones del estudio.

Aunque el trabajo que supone el desarrollo final de los puntos arriba recogidos no esté completamente terminado, lo hasta ahora realizado nos pone en el umbral del paso decisivo, es decir, del estudio empírico que motiva esta investigación.

\subsection{RECOGER LAS MUESTRAS DE LOS HNN}

Hemos procedido a recoger muestras de los textos que los alumnos han elaborado en sus clases, con el fin de determinar qué tipos de textos deben escribir, qué necesidades léxicas presentan y con qué dificultades se encuentran. Esta información es esencial para seleccionar las colocaciones más rentables en distintos niveles de competencia del español, desde B1.2 a C1.2, y ajustar la instrucción a los requisitos léxicos de los textos que han de redactar los aprendices. Asimismo podremos detectar errores en el uso del vocabulario que son habituales en las producciones no nativas e incluir en la secuencia didáctica actividades específicas que reduzcan su ocurrencia en los textos. 


\subsubsection{Perfil de los aprendices}

Contamos con las producciones de 24 alumnos: 20 estudiantes de movilidad Erasmus y de programas de Study Abroad que realizaron sus cursos en el semestre de otoño del 2017-2018 y 4 que realizaban el Máster oficial de la Universidad de Granada en Didáctica del español como lengua extranjera entre 2016-2018. De origen centroeuropeo, en su mayoría alemanes, 1 italiano, 2 serbios, 2 chinas. El nivel de lengua varía entre el B2.1 y el C1.1 e incluso C1.2, con algún alumno de origen hispano.

Los textos recopilados corresponden a 10 especialidades, entre las que predominan las asignaturas adscritas al área de Humanidades: Física, Ingeniería, Empresariales, Psicología, Antropología, Sociología, Lingüística, Traducción, Literatura y Didáctica del español como lengua extranjera.

En un primer momento no se van a tener en cuenta las variables en la producción que puedan suponer las diferencias de las L1 de los aprendices ni su área de especialidad, pues nos interesa fundamentalmente recabar información de carácter general sobre sus necesidades de aprendizaje y sobre las exigencias léxicas que les plantean los textos que deben redactar durante su formación universitaria.

\subsubsection{Tipología de textos}

Los textos que constituyen la muestra responden a distintas finalidades de la práctica docente. Se trata de fichas de clase, aplicaciones concretas de presupuestos teóricos, resúmenes de artículos, informes, proyectos y trabajos de investigación y presentaciones orales.

Los textos tienen diferente longitud y en ellos predominan las secuencias expositivas. Con vistas al desarrollo pedagógico enfocado en la escritura académica, sería útil llevar a cabo un análisis de las características textuales de cada modalidad, pero en los límites de nuestro trabajo no parece necesario por el momento, ya que lo más relevante es identificar un tipo de texto académico suficientemente general para que sea la fuente de la selección de las colocaciones y el modelo textual al que dirigir la práctica didáctica. 


\subsection{LA CREACIÓN DE UN CORPUS DE HN}

Teniendo en cuenta los textos que los estudiantes deben realizar hemos decidido restringir los análisis a un único género textual, que de hecho no se encuentra entre la lista de los escritos académicos reseñados en el epígrafe anterior. Nos referimos al abstract o resumen de investigación.

Varios han sido los motivos para seleccionar este género: los rasgos propios del texto, su extensión y la facilidad para acceder a él. En lo referente a los rasgos textuales hemos tomado en consideración que en un resumen de investigación se concentran una gran parte de las funciones discursivas que aparecen en otros escritos académicos: se presenta el tema que va a tratarse, se contextualiza el estudio con respecto a la corriente en que se inscribe, se expone una hipótesis u objetivo del trabajo o la estructura del artículo, etc. Por ello, en dichos textos se encuentra vocabulario variado apropiado a la expresión de cada una de dichas funciones.

Por lo que respecta a la extensión del resumen de investigación, el hecho de que su longitud oscile entre 200 y 300 palabras hace que sea un buen punto de partida para desarrollar la conciencia lingüística de los aprendices, ya que pueden analizarse varios ejemplos en cada sesión, abarcar su totalidad e identificar una estructura recurrente en ellos, que es independiente del tema concreto del estudio que presentan.

Por último, como apuntábamos, se trata de un modelo textual al que es fácil acceder. Podemos contar con corpus de resúmenes en las actas de congresos de distintas especialidades o en bases de datos como Teseo, en la que están registrados los resúmenes de las tesis doctorales presentadas en la universidad española.

Hemos seleccionado un corpus de 100 resúmenes de investigación, 5 por cada una de las especialidades que hemos incluido más arriba, con lo que consideramos que tanto los criterios de representatividad como los de generalidad y extensión están adecuadamente contemplados.

\subsection{DeLIMiTAR EL OBJETO DE ESTUdio}

El discurso académico no solamente se caracteriza por su objetividad, la precisión de sus términos y la especialización del vocabulario, sino también por una suerte de 
esqueleto de constructos lingüísticos esenciales a su desarrollo. Nos referimos con esto a los llamados paquetes léxicos ${ }^{5}$ y a las colocaciones.

La elección de las colocaciones como el tipo de segmento léxico en el que vamos a centrarnos puede justificarse por la mayor dificultad de su aprendizaje espontáneo por parte de los aprendices, lo que no es el caso de los paquetes léxicos, los cuales, debido al lugar preeminente que ocupan en la estructura del discurso son fácilmente detectables por los mismos aprendices. En efecto, el hecho de que la unión de los elementos de la colocación parezca diluirse en la fluidez de la frase difumina su carácter de constructo; el aprendiz suele pasar a través de ellas sin notar la frecuencia con la que una palabra sigue a la otra y aún menos la utilidad para la fluidez del discurso de que así ocurra. Las colocaciones cobran aún más importancia si se consideran desde el punto de vista productivo, ya que al no dominarlas el aprendiz puede notar la falta de fluidez de su discurso e incluso verse incapacitado para producirlo. Por otra parte, la ignorancia de la palabra concreta en la colocación - y esto tiene una especial relevancia para la precisión del lenguaje académico - suele llevar al aprendiz a sustituirla por un término que considere equivalente en su lengua materna o por otro término del nivel de español que sí domina, lenguaje cotidiano, por lo que en lugar de la precisión cae en la vaguedad de la expresión. Por ejemplo subrayar la importancia puede convertirse en marcar/acentuar o reafirmar la importancia, según la lengua materna del aprendiz.

En este trabajo, a efectos de la selección de los grupos léxicos, hemos consignado el verbo como elemento primordial de la colocación, ignorando en un principio su posición como base o como colocativo. Nos hemos centrado en las colocaciones de los siguientes tipos:
a) Nombre (sujeto) + verbo: agravarse una situación
b) Verbo + nombre (CD): abordar el tema
c) Verbo + preposición + nombre: centrarse en el estudio

\footnotetext{
${ }^{5}$ Los paquetes léxicos se definen como secuencias de más de dos palabras que aparecen en un registro determinado con una frecuencia mayor de la esperada (Biber et al., 1999). Se trata de expresiones formulaicas, del tipo a modo de introducción, en contra de estos argumentos o volviendo al asunto que nos ocupa, que tienen un papel destacado en la construcción del discurso.
} 
Incluiremos además otro tipo de colocaciones que resultan interesantes en los textos académicos como son las colocaciones de adverbios en -mente con adjetivos o adverbios, con las que se introducen las valoraciones que el autor quiere hacer explícitas en su discurso (explícitamente mencionado, verdaderamente interesante).

Aunque, en principio, no planeamos incoporar las colocaciones del tipo sustantivo + adjetivo (aspectos innovadores, numerosos ejemplos) o de sustantivo + preposición + sustantivo (una amplia variedad de posibilidades), la recopilación del material del corpus nos ha inducido a tratarlas en algunas ocasiones (centrarse en determinados aspectos).

Asimismo, pese a no tratarse de colocaciones, parece aconsejable no dejar de lado otras combinaciones léxicas, cuya ocurrencia es frecuente en los resúmenes de investigación en tanto que cumplen funciones textuales propias de este género. Por ejemplo: conseguir demostrar / lograr (perífrasis) con la función "indicar los objetivos del estudio".

\subsection{CREAR EL GLOSARIO DE COLOCACIONES}

Hasta el momento hemos consignado un número relativamente alto de colocaciones de ocurrencia frecuente en nuestro corpus, que consideramos suficiente hacia el fin hacia el que se dirige este trabajo, lo que no es óbice para que pueda ser ampliado si la puesta en práctica de los ejercicios diseñados así lo aconsejara.

Presentamos a continuación una limitada muestra de este glosario:

\begin{tabular}{|l|l}
\hline Abordar el tema & Favorecer las medidas \\
Abrir perspectivas & Fomentar el uso de... \\
Agravar[se] la situación & Garantizar el resultado \\
Apoyar la tesis & Impedir el aumento ... \\
Aumentar el número de... & Implicar el uso de... \\
Basarse en el estudio & Incluir [el estudio] \\
Centrarse en determinados aspectos & Mostrar [el estudio] \\
Comprobar los resultados & Motivar la investigación \\
Concluir [el estudio] & Plantear el tema \\
Considerar la importancia & Presentar los resultados \\
Conllevar consecuencias & Prestar atención a los factores \\
Deducir el significado & Pretende [el estudio] \\
Definir una palabra & Producirse cambios \\
Demostrar la teoría & Proporcionar una visión \\
Desarrollar una habilidad & Promover la aplicación \\
Describir el procedimiento & Provocar una respuesta \\
Destacar un aspecto & Referirse a [este término] \\
Determinar las razones & Reforzar los vinculos \\
\hline
\end{tabular}




\subsection{SISTEMATIZAR EL REPERTORIO LÉXICO}

Una vez recopilado un número significativo de combinaciones debemos encontrar modos de organizarlas para presentar la información del glosario de tal forma que sea fácilmente manejable y accesible para los usuarios.

Dado que el modelo textual elegido para la compilación del corpus es el resumen de investigación de artículos académicos, tesis doctorales y otros trabajos de investigación similares, el rango de colocaciones elegido se ve claramente inserto en contextos discursivos con funciones textuales como las siguientes: presentar el tema, contextualizarlo dentro del campo de investigación, justificar la importancia del estudio, señalar hipótesis y objetivos de trabajo, presentar la metodología seguida, anticipar conclusiones. El trabajo didáctico consistirá en poner de relieve la estructura discursiva del abstract para que los alumnos puedan apreciar la función que en él desempeñan las colocaciones y tenerlas disponibles en la elaboración de sus propias producciones.

Otras opciones de presentación podrían tener en cuenta las colocaciones de los sustantivos más frecuentes (p.e. tema, investigación o conclusión), o bien, las relaciones paradigmáticas de ciertos segmentos colocacionales (exponer las razones frente a las razones expuestas). Esperamos que un estudio detallado de las entradas del glosario permitirá encontrar nuevas alternativas de sistematización de las colocaciones que faciliten el diseño del material pedagógico y, en última instancia, favorezcan su adquisición.

\subsection{EL DISEÑO DE LAS SECUENCIAS DIDÁCTICAS}

Hasta donde sabemos no existen unidades didácticas destinadas a la enseñanza del vocabulario - en especial de las colocaciones rentables- en los discursos formales académicos; de ahí que para el propósito de nuestra investigación, nos veamos obligados a acometer esta tarea, apoyándonos en la información recabada sobre los textos de HNN, el corpus de resúmenes de investigación y el glosario que hemos recopilado. Nos serviremos igualmente de las directrices que en el campo de la 
didáctica se han formulado para facilitar la enseñanza del léxico ${ }^{6}$. (Higueras, 2006a; Ferrando, 2010 y 2017)

Higueras (2006a) expone una serie de principios metodológicos para la enseñanza de las colocaciones en las clases de español como LE, claramente derivados de los presupuestos del Enfoque Léxico, al que aludíamos al inicio del presente trabajo, y que serán la base pedagógica de la instrucción que va a recibir el grupo experimental.

Dichos principios consisten en:

1. Ofrecer una enseñanza tanto explícita como implícita de las colocaciones. Es importante enseñar explícitamente el concepto y llevar a cabo en clase actividades que desarrollen la conciencia de este fenómeno. Para favorecer el aprendizaje incidental hay que promover la lectura extensiva y enfatizar los beneficios para el aprendizaje que suponen las tareas de audición. En el caso que nos ocupa, el material que ofreceremos a los estudiantes consistirá en resúmenes de investigación de su propia especialidad.

2. Crear actividades dirigidas a fomentar el aprendizaje autónomo, trabajando con obras de referencia como diccionarios de colocaciones, diccionarios generales y de sinónimos, así como utilizando las posibilidades que ofrece internet: con corpus -Linguee, Bab.la, etc.- o concordancias, entre otros ${ }^{7}$. En las actividades que vamos a proponer también utilizaremos, como comentábamos más arriba, el corpus de resúmenes de investigación que hemos seleccionado y la base de datos Teseo para la consulta de tesis doctorales.

3. Enfocar el aprendizaje en las categorías de campo semántico, función comunicativa o macrofunción, para que la práctica de las colocaciones pueda darse en situaciones de interacción reales o creadas para el aula, pero con las condiciones adecuadas para un uso de la lengua significativo y natural.

4. Estimular la memorización de las colocaciones mediante diferentes tipos de actividades -juegos, elaboración de tarjetas, ejercicios estructurales, etc.- y

\footnotetext{
${ }^{6}$ En el mercado editorial han aparecido asimismo obras como los manuales de clase Bitácora (Difusión, 2011) y Uso interactivo del vocabulario y sus colocaciones más frecuentes (Edelsa, 2012) que proponen formas sugerentes de plantear el aprendizaje de las colocaciones.

${ }^{7}$ Para una revisión de corpus disponibles para el aprendizaje del español, véase Buyse (2017).
} 
enseñar a recogerlas de forma distinta a la convencional, es decir, en mapas léxicos, cajas de colocaciones, etc., en lugar de hacerlo en listas.

5. Promover el uso de todas las habilidades comunicativas para reforzar la adquisición de las colocaciones. Aunque la finalidad de las actividades que proponemos sea principalmente la de facilitar la producción de textos académicos escritos, también se prestará atención a la producción oral del lenguaje académico, tal y como puede desarrollarse, por ejemplo, en un debate.

6. Repetir el contenido de las secuencias didácticas, de modo que el aprendiz, al encontrarse con las mismas colocaciones en distintos contextos pueda afianzar su dominio de las mismas. La secuencia debe incluir tareas de toma de conciencia de la colocación, así como de indagación de las relaciones léxicas que esta establece con otras unidades, como también tareas de automatización en prácticas controladas y de producción abierta oral y escrita.

Todo esto en nuestro caso deberá estar específicamente enfocado a la enseñanza de las colocaciones frecuentes en el vocabulario académico, puesto que las anteriores indicaciones hacen referencia al tratamiento didáctico del lenguaje cotidiano. En efecto, hay que reseñar diferencias entre las colocaciones del lenguaje general y las colocaciones para fines específicos. Por lo que respecta a aquellas, los expertos (Lewis, 2000) aconsejan que la instrucción se centre, en primer lugar, en nombres con poco significado (tener/buscar/encontrar trabajo) y en verbos deslexicalizados (tener hambre/tiempo/dudas), pues son menos perceptibles en el discurso, pese a ser de uso común y tener gran capacidad expresiva, y en segundo lugar, en aquellas colocaciones que difieren entre la L1 y la L2, en tanto que se pueden anticipar los problemas que le causan al aprendiz (Higueras, 2006: 31). Sin embargo, las colocaciones de discursos especializados no parecen responden a los mismos parámetros: no son tan transparentes ni la expresión tiene el mismo grado de estabilidad que las de la lengua cotidiana, de ahí que para su enseñanza habrán de adoptarse otros criterios.

Una vez recogidos los principios generales en los que se basa la instrucción que proponemos pasamos a presentar algunas actividades concretas, como ejemplos de su aplicación. 


\section{ACTIVIDAD 1:}

A. Señala en este texto qué grupos de palabras te parecen bloques prefabricados.

Este artículo va a comentar algunos de los aspectos innovadores de la práctica de la evaluación por ordenador y en concreto, en línea, con sus ventajas y algunos problemas todavía no resueltos, y va a centrarse en la explicación del diseño y uso del nuevo examen de acreditación de español del Centro de Lenguas Modernas de la Universidad de Granada, administrado enteramente en línea a través de la web 2.0. A lo largo del artículo vamos a mostrar algunos aspectos que deben tenerse en cuenta en el diseño de este tipo de exámenes y a discutir los resultados del examen y de su utilización. En el texto se exponen las circunstancias de la puesta en marcha del eLADE en el año 2012, el proceso de diseño y producción en línea, los protocolos de validación y estandarización que se siguen, así como el desarrollo de la cuarta y más reciente convocatoria. El objetivo es tanto contribuir a la difusión del primer examen en línea de dominio de español como promover la máxima transparencia respecto a todos los procesos relacionados con los exámenes, en consonancia con lo establecido en los protocolos internacionales de diseño y validación de exámenes de lenguas.

B. Anota 7 de ellas que consideres útiles para redactar tus propios textos y coméntalas con tus compañeros.

La actuación de los estudiantes en esta actividad nos va a dar una idea de su capacidad para detectar elementos prefabricados en el discurso, lo que a su vez nos permitirá desarrollar en ellos la conciencia del concepto de colocación frente al de otras agrupaciones léxicas (paquetes léxicos o expresiones idiomáticas) que suelen aparecer en los textos académicos con los que trabajan.

\section{ACTIVIDAD 2:}

Completa la siguiente parrilla con grupos de palabras del texto anterior que se ajusten a los patrones que abajo se indican. Ayúdate con los ejemplos que aparecen a continuación.

- Nombre + verbo:

a. Nombre (sujeto) + verbo: ocurrirse una idea

b. Verbo + nombre: resolver un problema / discutir los resultados

c. Verbo + preposición + nombre: hablar de politica /

- Nombre + adjetivo: una idea asombrosa

- Nombre + de + nombre: la bolsa de la compra

- Verbo + nombre + adjetivo: aprender un idioma extranjero

- Verbo + adverbio: llorar amargamente

- Adverbio + adjetivo: completamente agotado

(Parrilla extraída de Gómez Molina, (2004b), completada con Koike (2001) 
La finalidad de este ejercicio es dirigir la atención del estudiante hacia determinados patrones léxicos, lo que nos permite hacer una presentación de manera indirecta de una tipología general de las colocaciones. El estudiante debe poder identificar dichos constructos siguiendo los modelos indicados.

ACTIVIDAD 3:

A. Busca con un compañero/a otra forma de expresar el significado de los verbos subrayados.

La lematización de unidades pluriverbales en el Diccionario cognitivo-combinatorio de arquitectura renacentista (Jacinto García)

En las últimas décadas 1. se han producido cambios muy profundos en el ámbito de la lexicografía, no solo respecto al modo de hacer diccionarios, sino también de concebir la propia obra lexicográfica. A esto 2. ha contribuido, sin lugar a dudas, el desarrollo de las tecnologías digitales, que permiten gestionar corpus lingüísticos inmensos y también elaborar diccionarios a la medida del usuario. El género lexicográfico 3. que refleja mejor estos cambios es el diccionario de especialidad. Como sostienen los autores de la teoría funcional de la lexicografía, como Henning Bergenholtz,..., entre otros, estas obras pueden cumplir, sobre todo, dos funciones principales: una comunicativa y otra cognitiva. Dichas funciones son las que 4. quiere desempeñar el futuro Diccionario cognitivo-combinatorio de arquitectura renacentista. Es cognitivo porque 5. pretende cubrir lagunas de conocimiento sobre arquitectura renacentista; y combinatorio porque además 6 . aspira a facilitar la elaboración de textos, ya sean originales o traducciones, mostrando las posibles combinaciones de unidades léxicas especializadas. Sobre este aspecto concreto versará esta comunicación.

B. Discutid en clase cuáles os parecen las mejores alternativas.

C. Anota cuáles son las palabras que forman un bloque con los verbos marcados.

- P.e. se han producido cambios

D. Comprueba la comprensión que tus compañeros tienen del resumen. Hazles preguntas sobre el texto anterior utilizando las expresiones en azul.

- P.e. ¿Cómo son los cambios que se han producido en el ámbito de la lexicografía?

E. Busca en Linguee tres ejemplos en que se use el verbo base de la combinación y que puedan serte útiles. Compáralos con tus compañeros.

- P.e: Se han producido pocos avances en esa dirección 
F. Señala cuál de las expresiones siguientes no tiene el mismo significado que la expresión original:

a. Se han producido cambios: han tenido lugar, ha habido, se han motivado, se han realizado...

b. La tecnología ha contribuido al cambio: ha influido en, ha favorecido, ha causado, ha evitado, ha provocado...

Con esta secuencia de actividades se persiguen varios objetivos: en primer lugar, favorecer la reflexión de los aprendices sobre el significado de ciertos verbos de uso frecuente en el discurso académico y estimularlos a buscar sinónimos o expresiones equivalentes, que amplíen su vocabulario y les ofrezcan un conocimiento cualitativo de ellas; en segundo lugar, hacerlos conscientes de qué colocativos siguen a ciertos verbos, insistiendo en la idea de la rentabilidad de la colocación para conseguir precisión en sus enunciados; en tercer lugar, propiciar el uso de las colocaciones en la producción oral, con lo que se refuerza el dominio que los aprendices tienen sobre ellas, y por último, llamar su atención sobre la importancia del manejo de obras de referencia, como diccionarios o corpus digitales, para solventar dificultades de expresión.

Para el grupo de control habremos de diseñar actividades que contengan el mismo input de partida que las del grupo experimental, pero en las que se proponga un tratamiento didáctico diferente, que no se sustente en los presupuestos del Enfoque Léxico, sino que, por el contrario, ponga el foco en nivel de la palabra y de las relaciones paradigmáticas, en lugar de hacerlo en el valor de los grupos léxicos.

\subsection{ELABORACIÓN DE LOS INSTRUMENTOS DE MEDIDA}

Una vez dados los pasos anteriores procederemos a la elaboración de un pretest y un postest para recoger los datos del experimento. Ambas pruebas tendrán una parte controlada, de reconocimiento de grupos léxicos, y otra abierta, que mida la presencia de colocaciones en la producción escrita de los aprendices. 


\subsection{ANÁlisis DE LOS RESULTAdAOS}

La comparación de los resultados de los aprendices de cada grupo en el pretest y postest nos permitirá responder a las preguntas de investigación y sacar conclusiones sobre el mayor o menor efecto asociado a cada una de las metodologías didácticas empleadas.

Como fácilmente puede advertirse la confirmación o refutación de las hipótesis es una tarea práctica que deja poco lugar a la subjetividad. Cuanto más sea esto así, más habremos acertado en la confección de las pruebas que hemos presentado, ya que nuestro trabajo tiene una vocación eminentemente práctica en la enseñanza en el aula, tarea que no solamente no debe subordinarse a las investigaciones teóricas sobre adquisición de lenguas, sino que debería ser el evidente fin que estas se propusieran.

\section{BIBLIOGRAFÍA}

Alonso Ramos, M. (Dir.) (2004). Diccionario de colocaciones del español. La Coruña. Disponible en: http://wwwdicesp.com [con acceso el 2 de febrero de 2018].

Boers, F. \& Lindstromberg, S. (2009). Optimizing the Lexical Approach to Instructed Second Language Acquisition. Nueva York: Palgrave Mac Millan.

Bosque, I.(Dir.) (2004). Redes. Diccionario combinatorio del español contemporáneo. Madrid: SM.

Buyse, K. (2017). Los corpus como herramientas de aprendizaje del léxico, en F. Herrera y N. Sans (Eds.), Cuadernos de didáctica. Enseñar léxico en el aula de español. Elpoder de las palabras. Barcelona: Difusión, pp. 121-140.

Buyse, K. \& González Melón, «El corpus de aprendices Aprescrilov y su utilidad para la didáctica de ELE en la Bélgica multilingüe». Disponible en: https://cvc.cervantes.es/ensenanza/biblioteca ele/asele/pdf/23/23 0025.pdf [con acceso el 2511-2017]

Calsamiglia, H. \& Tusón, A. (1999). Las cosas del decir. Manual de análisis del discurso. Barcelona: Ariel.

Ferrando, V. (2017). El papel de las colocaciones en la enseñanza y el aprendizaje del español, en F. Herrera y N. Sans (Eds.), Cuadernos de didáctica. Enseñar léxico en el aula de español. El poder de las palabras. Barcelona: Difusión, pp. 95-104.

Gledhill, C. (2000). The Discourse Function of Collocation in Research Article Introductions. English for Specific Purposes, 19, pp. 115-135.

Gómez Molina, J. R. (2004a). La subcompetencia léxico-semántica, en J. Sánchez Lobato e I. Santos Gargallo (Ed.), Vademécum para la formación de profesores. Madrid: SGEL, pp. 491-510. 
Gómez Molina, J. R. (2004b). Las unidades léxicas. Carabela, 56, pp. 27-50. Disponible en: https://dialnet.unirioja.es/servlet/articulo? codigo $=5305659$.

Higueras, M. (2004). Necesidad de un diccionario de colocaciones para aprendientes de ELE, en M. ${ }^{a}$ A. Castillo Carballo, O. Cruz Moya, J. M. García Platero y J. P. Mora Gutiérrez (Coords.), Las gramáticas $y$ los diccionarios en la enseñanza del español como segunda lengua, deseo y realidad: Actas del XV Congreso Internacional de ASELE. (2005) Sevilla. pp. 480-490. Disponible en: https://cvc.cervantes.es/ensenanza/biblioteca ele/asele/pdf/15/15 0478.pdf

Higueras, M. (2006). Las colocaciones y su enseñanza en la clase de ELE. Madrid: Arco Libros.

Hyland, K. (2008). As can be seen: Lexical bundles and disciplinary variation. English for Specific Purposes, 27, pp. 4-21.

Lewis, M. (1993). The Lexical Approach. The State of ELT and a Way Forward. Londres: Language Teaching Publication.

Lewis, M. (Ed.) (2000). Teaching collocation. Further Developments in the Lexical Approach. Boston: Thomson y Heinle.

Montolío, E (Coord), Figueras, C., Garachana, M. \& Santiago, M. (2000). Manual práctico de escritura académica. Barcelona: Ariel.

Otañi, I. (2005). El resumen o abstract del artículo de investigación en español: Características retóricolingüísticas, en G. Vazquez, (Coord), Español con fines académicos: de la comprensión a la producción de textos. Madrid: Edinumen, 45-72.

Pérez Serrano, M. (2015). Un enfoque léxico a prueba: efectos de la instrucción en el aprendiraje de las colocaciones léxicas. Tesis doctoral inédita. Universidad de Salamanca.

Thornbury, S. (2002). How to teach vocabulary. Harlow: Longman.

VV.AA. (2017). Enseñar léxico en el aula de español. El poder de las palabras. Barcelona: Difusión. 